\title{
Incidental Para-Nasal Polyps on Brain MRI Images in a Cameroonian Population
}

\author{
Dr. Uduma F. U. (Corresponding author) \\ Department of Radiology, Polyclinic Bonanjo, Douala \\ Department of Radiology, Abia State University Teaching Hospital, Aba, Nigeria \\ E-mail: felixuduma@yahoo.com \\ Dr Yaouda \\ Department of Otorhinolaringology, Polyclinic Bonanjo, Douala, Cameroon
}

Received: October 8, 2010 Accepted: October 26, 2010 doi:10.5539/gjhs.v3n2p118

\begin{abstract}
Rationale: Paranasal polyp is a soft tissue pear-shaped mass seen in the sinuses around the nostril. Allergy and inflammations are the major implicating factors. Small polyps could be of no concern to patients but large size could be a disturbance with perennial nasal obstruction and rhinorrhea. MRI with its superlative soft tissue contrast and multi-planarity facilitates the detection of paranasal polyps in brain MRI even when the referral is un-related to otorrhinolarygological features.Objective: To evaluate the prevalence and regional localisation of incidental polyps seen in brain MRI. Design and equipments: Prospective pioneer study using 0.3Tesla Hitachi AIRIS 11 MRI equipment. Setting: Polyclinic Bonanjo, Douala, Cameroon, a Specialist Hospital. Patients: 103 Patients referred for brain MRI from June 2009- Jan 2010 with only neurological symptomatology. Main outcome measured: Paranasal sinus was evaluated for polyps using T1W, T2W, FLAIR in different acquisitions. Results: 103 Patients were studied, 20 Patients had paranasal polyps with 14 (70\%) males and 6 (30\%) females forming a ratio of 2.33:1. Peak age range was 20-29years with 7 polyps (30\%) followed by 50-59years with $25 \%$. Polyps were rare in extremes of age. These 20 patients had 23 polyps due to 2 cases of bilateral maxillary polyps and single case of multiple polyps. The highest number of paranasal polyps 17 (73.91\%) were in the maxillary sinus, followed by sphenoidal and frontal sinuses. Majority of polyps were pedunculated and $<2 \mathrm{~cm}$. Conclusion: Paranasal polyps are easily detected by MRI. The highest location is in the maxillary sinus with male preponderance.
\end{abstract}

Keywords: Polyps, MRI, Paranasal sinus

\section{Introduction}

Paranasal sinuses are the 4 pairs of air containing cavities located around the nostrils. This consists of frontal, maxillary, ethmoidal and sphenoidal sinuses. Paranasal sinuses (PNS) play roles in humidifying inhaled air and reducing the weight of skull, though accurate function of paranasal sinuses is still unclear. PNS polyps are abnormal fleshy outgrowths from the mucosa of paranasal sinuses, usually inflammatory.

Though plain radiography is still the commonest tool to investigate PNS, but the sectional images of CT and MRI are more sensitive in yielding PNS polyps. CT has the advantage in demonstrating bony abnormalities while MRI has good soft tissue contrast. This enables polypoidal masses in PNS to be detected as an incidentalomas in patients who were referred for brain MRI studies for problems un-related to ear, nose, throat (ENT).

\section{Aim}

To investigate the prevalence of incidental Paranasal polyps shown on brain MRI scans performed primarily for neurological reasons

\section{Material and methods}

Prospective consecutive study of 103 patients who were referred for brain MRI in Polyclinic Bonanjo, Douala from June 2009 to January 2010 was done. The scans were done with 0.3Tesla HITACHI AIRIS 11 MRI 
machine. Technical factors used were T1W, T2W and FLAIR. Important acquisitions were coronal, axial and sagital. Regional localisation was done using sagital and coronal acquisitions as screening tool. Enhanced images with Gadolinium pentetate dimeglumine were also used in evaluations.

Inclusion criterion is referral for neurological signs and symptoms. Exclusion criteria are referrals related to ENT. Mucosal retention cysts were excluded because they are T1W and T2W hyperintense. Differentiating them from this is in contrast to polyps that are intermediate on $\mathrm{T} 1 \mathrm{~W}$ and hyperintense on $\mathrm{T} 2 \mathrm{~W}$.

Statistical analysis was done using SSPS 13.0 software

\section{Results}

Of the 103 patients studied, 59 were males and 44 females. Age range was 16 to 81 years, mean age 48.5 years. In all, 20 patients constituting 19.41\% of studied population had PNS polyps in one or more PNS. Peak age range was $20-25$ years with $30 \%$ followed by $50-59$ years with $25 \%$. Polyps were seen to be rare in extremes of age.

Of the 20 patients with polyps, 14 (70\%) were males and 6 (30\%) females, with male to female ratio of 2.33: 1 . These 20 patients had total number of 23 PNS polyps, with the highest number of 17 polyps (73.91\%) located in the maxillary sinuses, and the least $2(8.78 \%)$ located in the frontal sinuses. Sphenoidal polyps were 4 (17.39\%) and no ethmoidal polyp was seen. Male-female ratio of 3.25:1 was seen in maxillary sinus polyps, 2:0 in frontal sinus polyps and 1; 1 in sphenoidal sinus polyps. Polyps less than $2 \mathrm{~cm}$ in greatest diameter were 17 (72.72\%) and 2-5cm polyps were $6(27.28 \%)$. 2 patients had bilateral maxillary polyps while 1 patient had multiple polyps. Sessile polyps with broad base were 4 (17.4\%), the rest $82.6 \%$ were pedunculated.

13 males (76.47\%) and 4 females (23.53\%) had, maxillary polyps with male to female ratio of 3.25:1. Right maxillary polyps were $11(64.70 \%)$ and $6(35.30 \%)$ were left with ratio 1.33:1, the highest incidence of 7 cases were seen at 20-29years, followed by 50-59years with 4 cases. Maxillary polyps in this study commonly arose from the floor (5 polyps), followed by 3 polyps each from the roof, medial and lateral walls. Bilateral maxillary polyps were seen in 2 patients who were 21year-old male male and 22year-old-female. 1 case of multiple polyps in a 43year old man with polyposis in left frontal, maxillary and sphenoid sinuses was seen. Polyps associated with rhino-sinusitis were 9 , proptosis - 1 , retention cyst -1

\section{Discussion}

Though the symptomatology of paranasal polyps is perennial nasal obstruction, anosmia/ hyposmia, rhinorrhea and sneezing, but many are asymptomatic especially small polyps. MRI allows better differentiations of soft tissue structures within the sinuses. PNS polypoidal masses are readily seen in brain MRI studies with enough information for affirmative regional localization due to MRI multi-planarity.

Nimbkar and Sane in their histochemical study of 70 polyps specimen, concluded that $77.2 \%$ were due to allergy and infection while $22.8 \%$ were non-allergic in origin. They therefore classified polyps into 5 histiological groups namely oedematous polyps, fibrous polyps, glandular or cystic polyps, Angiectatic polyps and mixed types. This is based on its components as argued by different authors. Virchow and his pupils believed polyps are myxomatous tumours, Eggston and Wolff view it as passive mucosal oedema, Anderson and Bing's thought it as proteinacous exudates while Weisskopf and Burns consider it mucopolysacharides. Whatever, allergy, infection and inflammations are paramount. The classic triad of symptoms (Samter's triad) exist in patients with aspirin induced bronchial asthma, Aspirin (ASA) sensitivity and chronic rhino-sinusitis with subsequent formation of nasal polyps. This raises nasal /paranasal polyps prevalence up to $70 \%$ in ASA-intolerant patients unlike $4 \%$ of nasal polyps in normal populace. Infective origin of paranasal polyps like invasive aspergillosis involving multiple PNS have been reported by Agarwal, et al.

In our study, the majority of the polyps were single and predominantly maxillary. Individual polyps could be antral, choanal polyp, a benign massive polyp or any benign or malignant tumour. In general, multiple benign polyps are seen in Bronchial Asthma, Cystic fibrosis (CF), Allergic Rhinnitis, Allergic fungal sinusitis (AFS), Chronic Rhino-sinusitis, ASA intolerance, Alcohol intolerance, Cililary Dyskinesia, Kartagener's Syndrome, Churg - Strauss Syndrome, Young Syndrome (chronic sinusitis, nasal polyposis, Azospermia), Non-allergic rhinitis with eosinophilia syndrome (NARES). Unlike antro-choanal polyps, spheno-choanal polyps are rare. Choanal polyps are solitary benign tumours arising from one paranasal sinus and pass over drainage ostium of this sinus projecting itself to the choana up to the naso-pharynx.

The prevalence rate of $19.41 \%$ in our study agrees with US prevalence range of $0.2-28 \%$ with incidence rate among adults of 1-4\%. Also male predominance of 2.33:1 agrees with 2-4:1 in adult Americans. No case of PNS polyps was seen in this study in patients below 10years as in other studies where polyps are rare under age 5 . 
Manifest starts at 20years and common in those older than 40years. The peak age in this study was however $20-29 y e a r s$ and followed closely by $50-59 y e a r s ~(25 \%)$. In our study, small polyps $<2 \mathrm{~cm}$ in maximum diameter predominates with $72.72 \%$. This is advantageous as they are less symptomatic and more responsive to medical treatment like steroids. But the larger polyps though more detectable with anterior rhinoscopy and nasal endoscopy but are more commonly associated with systemic diseases like CF. Jorrissen, et al. detected polyps in $56 \%$ of CF patients, $17 \%$ of them had small polyps while the remaining $39 \%$ had larger polyps. Such larger polyps require evaluation with CT and MRI. In this study, majority of the polyps were pedunculated. Differentiations of nasal polyps from mucous retention cyst are usually difficult but the retention cyst is hyperintense on T1W and T2W due to its muco-proteinous content unlike polyp which is T1W intermediate and T2W hyperintense.

PNS polyps are amenable to medical and surgical treatment. Medical treatment is steroid therapy. Surgical managent ranges from polypectomy to intranasal ethmoidectomy. But the high recurrence after polypectomy led to development of Functional Endoscopic Sinus Surgery with recurrence reduced to just 10\%.

Differential diagnoses of paranasal polyps are Encephaloceles, Glioma, Haemangiomas, Papillomas, Juvenile Nasopharyngeal angiofibroma, Rhabdomyosarcoma, Lymphoma, Neuroblastoma, Sarcoma, Chordoma, Inverting Papilloma, Cystic Fibrosis (CF), Chronic Sinusitis, and Allergic Fungal Sinusitis.

\section{Conclusion}

The significance of this study are summarised thus:

Paranasal (PNS) polyps are readily evaluated with MRI

PNS polyps in Douala, Cameroon are on the high side of documented prevalence rate of some countries.

The commonest affected sinus is maxillary sinus, followed by sphenoid, frontal and ethmoid sinuses in decreasing order

Pedunculated polyps are more common than sessile polyps

\section{References}

Agarwal, S., Kanga, A., Sharma, V., Sharma, D. R., \& Sharma, M. L. (2005). Invasive aspergillosis involving multiple paranasal sinuses, a case report. Indian Journal of Medical Microbiology, 23 (3), 195-197. http://dx.doi.org/10.4103/0255-0857.16595

Bachert, C., Waterlet, J. B., Geraert, P., \& Van Cauwenberge, P. (2005). Pharmacological management of nasal polyposis. Drugs, 65 (11), 1537-1552. http://dx.doi.org/10.2165/00003495-200565110-00006

De Jesus, E. P. F., Goto, E. Y., Marone, S. A. M., Zagati, L., Haddad, A., \& Portes, K. P., (2005). Spheno-choanal Polyp Case Report and Literature Review. International Archives of Otorhinolaryngology, 9 (1).

Gulati, S. P., Anshu, M. S., Wadhera, R., \& Deeo, A. (2007). Efficacy of Functional Endoscopic Sinus Surgery in the treatment of Ethmoidal Polyps. The Internet Journal Otorrhoinolaryngology, 7 (1).

Hiari, M. \& Hiari, M. A. (1998). Incidental Paranasal Sinus Inflammatory Changes in a Jordanian Population. Eastern Mediterranean Health Journal, 4 (2), 308-311.

Jorrissen, M. B., De. Boeck, K., \& Cuppens, H. (1999). Geneotype - Phenotype Correlations for the paranasal sinuses. American Journal of Respiratory and Critical Care Medicine, 159, 1412-1416.

Kowalski, M. L., Bienkiewicz, B., Pawliczak, R., \& Kordek, P. (2003). Nasal Polyposis in Aspirin -Hypersensitive Patients with Asthma (Aspirin Triad) and Aspirin Tolerant Patients. Journal of the World Allergy Organisation, 15 (6), 246-250. http://dx.doi.org/10.1027/0838-1925.15.6.246

Maempel, Z., Hide, I. G., \& Hill, J. (2002). A hearing problem with a difference. British Journal of Radiology, 75, 85-86.

McClay, J. E. \& Nasal Polyps (2006). E-Medicine, Oct 22.

Mendes, I. \& Elaine, A. (2004). Evaluation of Inter-observer agreement level in Sinonasal polyposis though Computed Tomography Analysis. BJORL, 504-510.

Nimbkar, S. A. \& Sane, S. Y. (1978). Histiology and Histochemistry of Nasal Polyps. JPGM, 24 (4), 231-234.

Okuyemi, K. S. \& Tsue, T. T. (2002). Radiological Imaging in the Management of Sinusitis. American Family Physician, 66 (10), 1882-1887.

Szczekik, A. \& Nizankowska, E. (2000). Clinical features and Diagnosis of Aspirin induced asthma. Thorax, 55 
(Suppl 2), 42-44. http://dx.doi.org/10.1136/thorax.55.suppl_2.S42

Yasuda, M., Niisato, N., Miyazaki, H., Hama, T., et al. (2007). Epithelial Ion Transport of Human Nasal Polyp and Paranasal Sinus Mucosa. American Journal of Respiratory Cell and Mollecular Biology, 36, 466-472. http://dx.doi.org/10.1165/rcmb.2006-0064OC

Table 1.

\begin{tabular}{|c|c|c|c|}
\hline \multicolumn{2}{|c|}{ Studied Population } & \multirow[b]{2}{*}{ Female } & \multirow[b]{2}{*}{ Total } \\
\hline Age Range & Male & & \\
\hline 0-9 & 1 & 3 & 4 \\
\hline $10-29$ & 7 & 4 & 11 \\
\hline $30-39$ & 6 & 5 & 11 \\
\hline $40-49$ & 8 & 11 & 19 \\
\hline 50-59 & 12 & 11 & 23 \\
\hline 60-69 & 15 & 8 & 23 \\
\hline 70-79 & 6 & 1 & 7 \\
\hline 80-89 & 1 & 1 & 2 \\
\hline \multirow[t]{2}{*}{ 90-99 } & 3 & $\mathbf{0}$ & 3 \\
\hline & $\mathbf{0}$ & $\mathbf{0}$ & 9 \\
\hline Total & 59 & 44 & 103 \\
\hline
\end{tabular}

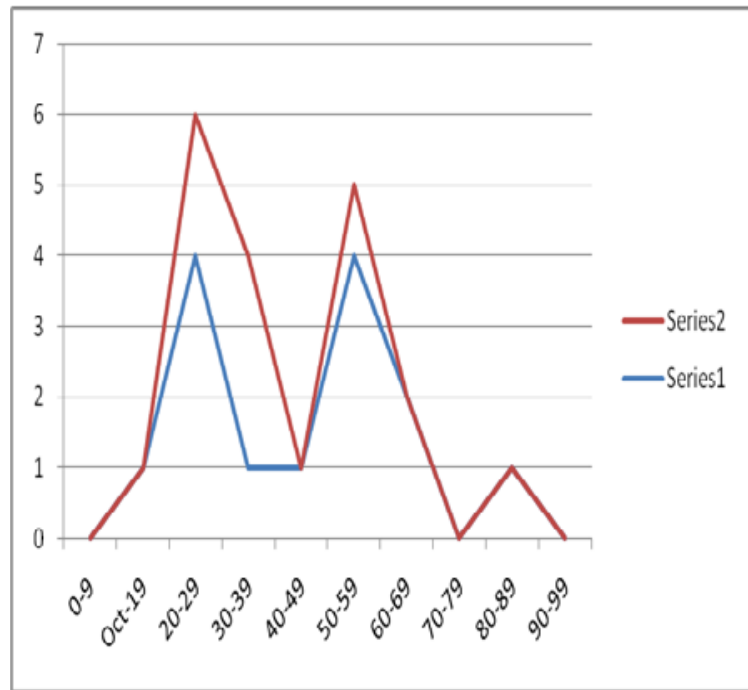

Figure 1. Age -Sex Distribution of Studied Population Seris $1=$ Females Series $2=$ Males

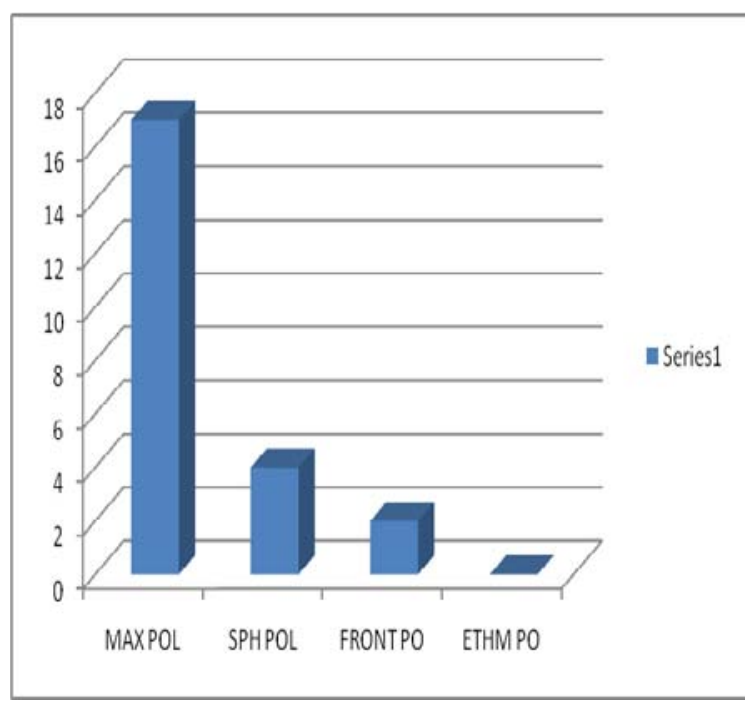

Figure 2. Regional Localisation of Polyps 


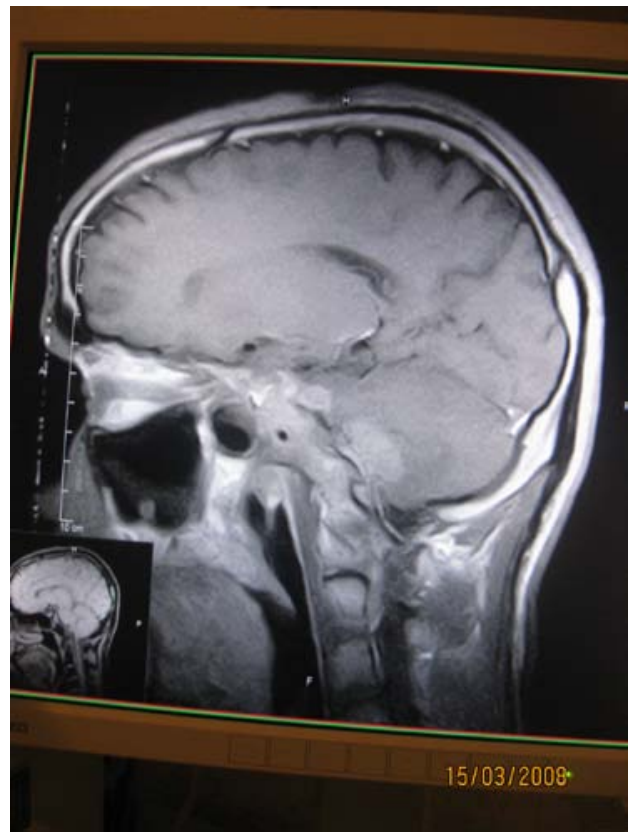

T1W.

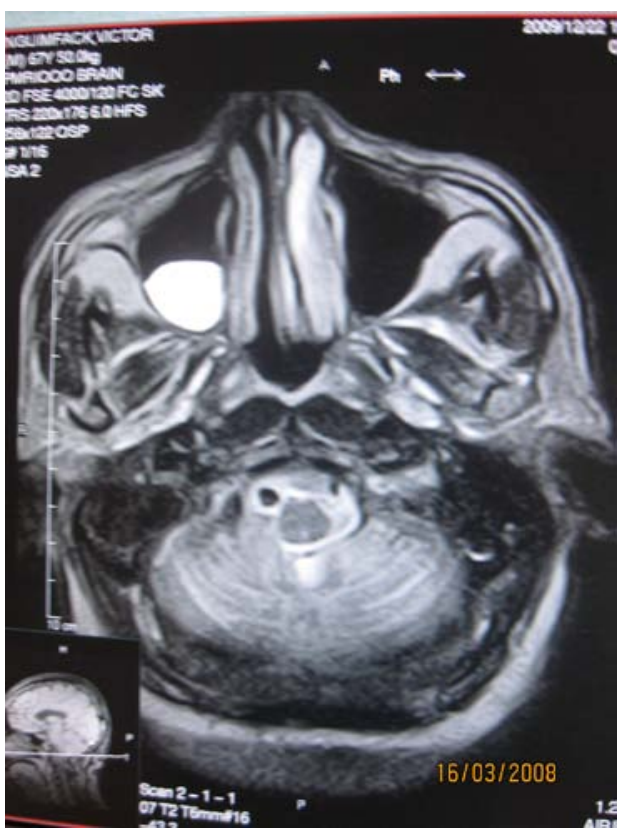

T2W.

Figure 3. showing right maxillary floor polyp 